\title{
Effects of epidural analgesia on colonic motility and patients' outcome in colorectal surgery: a retrospective study
}

\author{
Yesim Cokay Abut ${ }^{1}$, Ismail Okan², Hayal Tezer Kartal' ${ }^{1}$, Melih Ozdamar ${ }^{1}$, Tayfun Aldemir ${ }^{1}$ \\ 1Department of Anesthesiology, Vakif Gureba Training Hospital, Istanbul, Turkey \\ 2Department of Surgery, Vakif Gureba Training Hospital, Istanbul, Turkey
}

Przegląd Gastroenterologiczny 2011; 6 (5): 299-303

DOI: $10.5114 /$ pg.2011.25378

Key words: epidural, colonic surgery, colonic motility, postoperative ileus.

Address for correspondence: Yesim Cokay Abut MD, Fatma Sultan Mah. Kahal Bagi Sok. No: 46 Da: 3, Fatih-Istanbul 34093, Turkey, phone: 9021252321 51, fax: 9021262175 80, e-mail: yesimabut2000@yahoo.com

\begin{abstract}
Aim: To explore the hypothesis that epidural medication stimulates colonic motility, shortens hospital stay and causes fewer complications than general anaesthesia alone, we conducted a retrospective study on patients undergoing colorectal surgery. Material and methods: Sixty-two adult patients who underwent colectomy or proctocolectomy at Vakif Gureba Training Hospital between June 2006 and December 2008 were divided into two groups retrospectively: an general anaesthesia only group (group 1) and a combined epidural and general anaesthesia group (group 2). First faecal and gas discharge time, first mobilization time, length of hospital stay and postoperative complications in 30 days after surgery were obtained from patients' medical records.

Results: Between the patients in the two groups matched and found similar for preoperative characteristics, there were no differences for gas and faecal discharge time, mobilization time, length of hospital stay or complications.
\end{abstract}

\section{Introduction}

Postoperative ileus, a temporary inhibition of gastrointestinal function, plays a major role in postoperative morbidity and mortality, especially in abdominal surgery. Under normal conditions, tonic inhibitory sympathetic control dominates in gastrointestinal motility. Thus, blockade of splanchnic nerves with epidural local anaesthetics increases parasympathetic activity, so motility increases and perhaps the development of ileus decreases, theoretically.

To explore the hypothesis that epidural medication stimulates colonic motility, thereby shortening hospital stay and causing fewer complications than general anaesthesia alone, we conducted a retrospective study in patients undergoing colorectal surgery.

\section{Material and methods}

The study population consisted of 62 patients (39 men, 23 women), with ASA 2-3 status, who underwent colorectal surgery at Vakif Gureba Training Hospital between June 2006 and December 2008 (Tables I-III). Twenty-three patients were anaesthetized with general anaesthesia alone and conventional postoperative pain therapy, including both intravenous tramadol infusion and intramuscular non-steroidal anti-inflammatory drugs (group 1). The remaining 39 patients were anesthetized with general anaesthesia and epidural analgesia (group 2). In this group, local anaesthetics and fentanyl were given as intermittent injections via an epidural catheter $(n=12)$ or epidural PCA $(n=27)$ with similar doses. The same team of surgery and anaesthesiology performed all operations.

\section{Anaesthetic management}

All patients received general anaesthesia consisting of thiopentone (3-7 mg/kg), $1 \mu \mathrm{g} / \mathrm{kg}$ fentanyl, rocuronium and $1 \%$ end tidal sevoflurane, nitrous oxide, and oxygen (50\%/50\%). Intraoperative analgesia was established with intravenous fentanyl in group 1 and with epidural local anaesthetic $0.125 \%$ (bupivacaine or levobupivacaine) and $20 \mu \mathrm{g}$ fentanyl mixture 
in group 2. In group 2 epidural patients controlled analgesia consisting of $0.125 \%$ local anaesthetic and $0.20 \mu \mathrm{g}$ fentanyl, with bolus dose of $4 \mathrm{ml} / \mathrm{h}$, basal infusion rate of $4 \mathrm{ml} / \mathrm{h}$ and lockout interval $30 \mathrm{~min}$, were administered for 27 patients. The same epidural mixture was injected as intermittent bolus doses via an epidural catheter when postoperative VAS score was up to 3 for 12 patients in group 2. Epidural administration of local anaesthetics was started intraoperatively, and continued for up to $24 \mathrm{~h}$ postoperatively. On the second day of surgery, postoperative analgesia was was prescribed by the surgical team with both intravenous tramadol infusion and non-steroidal antiinflammatory analgesics.
Patients' first faecal and gas discharge time, first mobilization time, length of hospital stay, and postoperative complications in 30 days after surgery were obtained from patients' medical records. First mobilization time was determined as walking with help in their room. The hospital discharge criterion was determined when patients were fully mobile without assistance, tolerant to solid food, in the absence of infection and pain, and with passage of stool and gas. Postoperative complications recorded were intra-abdominal sepsis, anastomotic leakage, wound infection, pulmonary embolus, pneumonia, cardiac problems, cerebrovascular accidents, paralytic ileus, postoperative hypotension (in the first $24 \mathrm{~h}$ ) and epidural catheter related complications.

Table I. Demographic characteristics, type of surgery and clinical data of the two groups

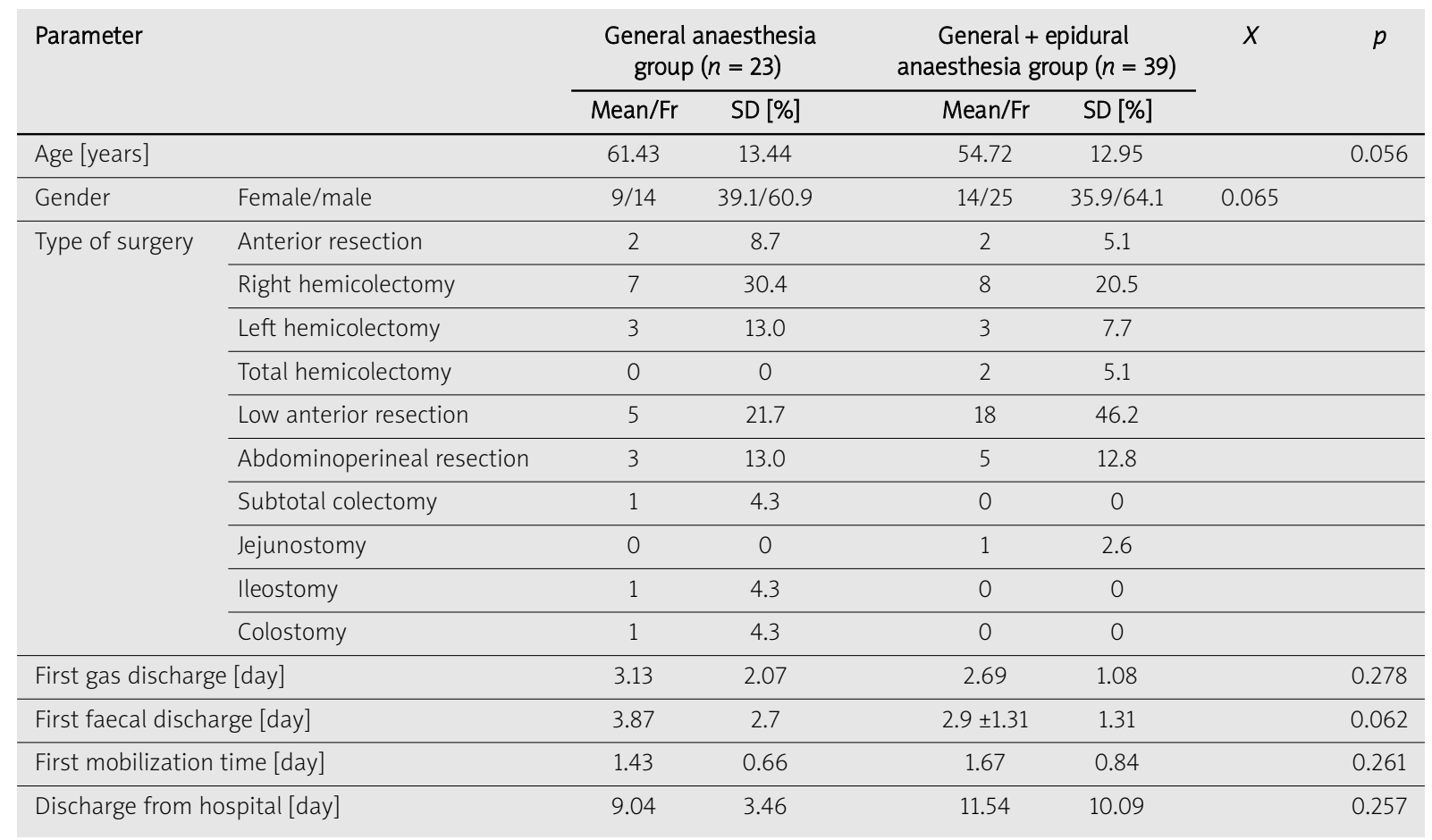

SD - standard deviation, Fr-frequency

Table II. Group 1 was compared with PCA group (27 patients in group 2) regarding first gas and faecal discharge and mobilisation time; there was no statistically significant difference between groups

\begin{tabular}{|c|c|c|c|c|c|c|}
\hline & \multicolumn{2}{|c|}{ First gas discharge [day] } & \multicolumn{2}{|c|}{ First faecal discharge [day] } & \multicolumn{2}{|c|}{ Mobilisation time [day] } \\
\hline & $\begin{array}{l}\text { In the first } \\
2 \text { days }\end{array}$ & Other days & $\begin{array}{l}\text { In the first } \\
2 \text { days }\end{array}$ & Other days & $\begin{array}{l}\text { In the first } \\
2 \text { days }\end{array}$ & Other days \\
\hline General anaesthesia $(n=23)$ & 11 & 12 & 8 & 15 & 21 & 2 \\
\hline $\begin{array}{l}\text { General anaesthesia and postoperative PCA } \\
\text { with local anaesthetic }(n=27)\end{array}$ & 14 & 13 & 14 & 13 & 25 & 2 \\
\hline Value of $p$ & \multicolumn{2}{|c|}{0.776} & \multicolumn{2}{|c|}{0.354} & \multicolumn{2}{|c|}{0.867} \\
\hline
\end{tabular}

$p<0.05$ is significant 
Table III. Postoperative complications in group 1 and group 2

\begin{tabular}{lccc} 
Complication & $\begin{array}{c}\text { General anaesthesia } \\
(n=9)(39.13 \%) \mathrm{Fr}(\%)\end{array}$ & $\begin{array}{c}\text { Epidural + general anaesthesia } \\
(n=14)(35.89 \%) \mathrm{Fr}(\%)\end{array}$ & Value of $p$ \\
\hline Postoperative hypotension & $2(8.69)$ & $6(15.38)$ & 0.799 \\
\hline Anastomotic leak & $0(0)$ & $2(5.12)$ & $3(7.69)$ \\
\hline Wound infection & $3(13.04)$ & $1(2.56)$ \\
\hline Pneumonia & $0(0)$ & $1(2.56)$ \\
\hline Bleeding & $0(0)$ & $0(0)$ \\
\hline Atrial fibrillation & $1(4.34)$ & $0(0)$ \\
\hline Cerebrovascular accident & $1(4.34)$ & $1(2.56)$ \\
\hline Intra-abdominal septicaemia & $0(0)$ & $0(0)$ \\
\hline lleus & $2(8.69)$ & $0(0)$
\end{tabular}

\section{Statistical analysis}

Data of the two groups were compared by $\chi^{2}$ test and independent $t$ test in NCSS 2007 software. Values of $p$ less than 0.05 were considered statistically significant.

\section{Results}

There was no difference between the two groups (group 1 and group 2) with regard to age, gender or type of surgery. Mean age was $61.43 \pm 13.44$ in the general anaesthesia group (group 1) and $54.72 \pm 12.95$ in the general anaesthesia + epidural analgesia group (group 2); the difference was not significant.

Gas and faecal discharge time, number of postoperative complications, length of hospital stay and mobilization time were similar between the two groups. First gas and faecal discharge time were earlier in the epidural + general anaesthesia group, but no significance was noted. Although it did not reach significance, discharge from hospital was longer in group 2.

However, the complication rate between groups was similar. Differences between the two groups in discharge from hospital time and complication rate were statistically not significant. There was only one case of death in the postoperative period, because of intraabdominal septicaemia in group 2 . This patient was an HIV virus transporter and immunosuppressants might have played an important role in the patient's outcome.

When the postoperative epidural intermittent injection group (about 12 patients in group 2) was excluded and group 1 was compared with the PCA group (about 27 patients in group 2), no statistically significant difference was found regarding first gas and faecal discharge, and mobilization time on the first 2 days of surgery than other days.

\section{Discussion}

All abdominal operations cause some degree of postoperative ileus. Abdominal pain, stress of surgery, excessive handling of the bowel, electrolyte imbalance and systemic opioids prolong ileus by diminishing propulsive contractions. It is known that epidural anaesthesia produces sympathetic blockade at the level of the spinal cord. When thoracic and lumbar sympathetic nerves are blocked by epidural analgesia, there is a consequent, relative increase in parasympathetic tone on gastrointestinal activity and, theoretically, this parasympathetic hyperactivity increases gastrointestinal motility [1].

Epidural analgesia has commonly been used in association with major surgery to improve patient outcome since 1900 [2], and it has many positive effects on mobilisation, ventilatory function and pain treatment [3-5]. It has been suggested that postoperative epidural analgesia is associated with an increase in colonic motility and earlier return of gut function. First, Udassin et al. showed that epidural anaesthesia accelerates the recovery of post-ischemic bowel motility in rats [6]. In 1995, Morimito reported that epidural analgesia with fentanyl shortens postoperative ileus after proctocolectomy operations [7]. Since then, many randomized clinical trials have been published with different results regarding the effect of epidural anaesthesia on postoperative ileus or colonic motility [8-10].

To explore the hypothesis that epidural medication stimulates colonic motility, shortens hospital stay and causes fewer complications than general anaesthesia alone, we conducted a retrospective study with the same operation by a single team of surgery and anaesthesiology.

In clinical practice, combination of an opioid with a local anaesthetic is the most commonly used for 
epidural analgesia. Addition of an opioid allows adequate analgesia and less adverse effects of local anaesthetics, such as hypotension and motor deficit [11, 12]. Therefore, we used epidural bupivacaine or levobupivacaine and fentanyl mixture for epidural analgesia. We prefer fentanyl to morphine because of morphine's wellknown adverse effects on colonic motility $[13,14]$.

The influence of postoperative local anaesthetics on anastomotic healing remains unclear. Increased splanchnic blood flow may aid anastomotic healing and reduce leakage. Anastomotic leak rates in clinical studies are similar after epidural blockade and general anaesthesia [15-17]. For example, a meta-analysis of 562 patients from randomized trials reported between 1966 and 2000 did not detect a significant difference in anastomotic leak between patients receiving either postoperative epidural local anaesthetic or local anaestheticopioid combinations, or those receiving either systemic or epidural opioid [18]. In our study, we had different results. We had 2 cases (5.12\%) in which anastomotic leak developed, in the epidural + general anaesthesia group (group 2).

There are many studies comparing postoperative epidural analgesia with systemic analgesia. However, since 1977, there is no consensus about the location of the epidural catheter. In early studies, the location of the epidural catheter was not specified in the literature $[7,19,20]$. In some of the studies with epidural catheter placement above T12, gastrointestinal function recovered more rapidly when epidural analgesia was used than when patients received systemic analgesics. Nevertheless, there are some concerns about thoracic epidural analgesia [15]. Studies in which the epidural catheter was positioned at or below $\mathrm{T} 12$ were equally as likely to show faster recovery of gastrointestinal function with epidural analgesia as systemic analgesia $[16,21]$. It seems to be that thoracic epidural blockade is more effective than lumbar epidural blockade in reducing the duration of ileus; however, the benefit or the clinical importance of 12 -h or earlier rapid gas or faecal discharge time is debatable. We observed that L1-2 catheter location ensures sensory block level at T4, and this is enough for postoperative analgesia. Besides, there was no case in which systemic analgesia associated with more rapid recovery of gastrointestinal motility.

Duration of administration of epidural analgesia after operation was almost $24 \mathrm{~h}$ in our study because of the practical difficulties of maintaining an epidural infusion 'on the ward' during patient mobilization, especially in fast track surgery. Despite the benefits of postoperative analgesia for the patient's well-being, studies have not demonstrated a shorter hospital stay with epidural analgesia. For example, Lehman and Wiseman
[19] reviewed the hospital courses of 102 patients who underwent elective colonic surgery. All patients received general anaesthesia; 41 patients received postoperative epidural analgesia. There were no significant differences in duration of ileus or length of hospital stay in this retrospective study. It is similar with our results. Interestingly, discharge from hospital was longer in group 2.

The duration of ileus after gastrointestinal surgery is assessed in various ways. They include measurement of the interval from operation to passage of gas or faecal discharge, the transit of barium on serial radiograms, and clinical variables such as time to removal of the nasogastric tube [22, 23]. Patients' first faecal and gas discharge time were obtained from patients' medical records retrospectively in our study. The nasogastric tube was removed by surgeons for all patients, on the first postoperative day, because our surgery clinic has preferred to perform "fast track colonic surgery".

The practical difficulties of both maintaining an epidural infusion 'on the ward' during patient mobilization, intermittent local anaesthetic administration, timing of epidural infusion ( $24 \mathrm{~h}$ may not be enough) or the level of the catheter were probably limited in our study results. However, it was mentioned that systemic analgesia was not associated with more rapid recovery of gastrointestinal motility than epidural analgesia. We think that additional studies are required to determine the ideal PCA regimes, optimal timing of administration and level of epidural blockade causing fewer complications in gastrointestinal surgery.

\section{References}

1. Steinbrook RA. Epidural anaesthesia and gastrointestinal motility. Anesth Analg 1997; 86: 837-44.

2. Tuffier TH. Anesthesie medullaire chirurgicale par injection sous-arachno lombaire de coca; technique et resultats. La Semaine Medicale 1900; 20: 167-9.

3. Liu S, Carpenter RL, Neal JM. Epidural anaesthesia and analgesia: their role in postoperative outcome. Anesthesiology 1995; 82: 1474-506.

4. Jorgensen H, Fomsgaard JS, Dirks J. Effect of epidural bupivacaine vs combined epidural bupivacaine and morphine on gastrointestinal function and pain after major gynaecological surgery. BJA 2001; 87: 727-32.

5. Jayr C, Thomas H, Rey A, Farhat F. Postoperative pulmonary complications. Anesthesiology 1993; 78: 666-76.

6. Udassin R, Eimerl D, Schiffmann J, Haskel Y. Epidural anesthesia accelerates the recovery of postischemic bowel motility in the rat. Anesthesiology 1994; 80: 832-36.

7. Morimito H, Cullen JJ, Messick JM Jr, Kelly KA. Epidural analgesia shortens postoperative ileus after ileal pouch-anal canal anastomosis. Am J Surg 1995; 169: 79-83.

8. Schnitzler M, Kilbride MJ, Senagore A. Effect of epidural analgesia on colorectal anastomotic healing and colonic motility. Reg Anesth 1992; 17: 143-7. 
9. Treissman DA. Disruption of colonic anastomosis associated with epidural anesthesia. Reg Anesth 1980; 5: 22-3.

10. Bigler D, Hjortso NC, Kehlet $H$. A case of disruption of colonic anastomosis two hours post-operatively during continuous epidural analgesia. Anaesthesia 1985; 40: 278-80.

11. Aromaa U, Lahdensuu M, Cozanitis DA. Severe complications associated with epidural and spinal anaesthesias in Finland 1987-1993. A study based on patient insurance claims. Acta Anaesthesiol Scand 1997; 41: 445-52.

12. Shafer AL, Donnely AJ. Management of postoperative pain by continuous epidural infusion of analgesics. Clin Pharm 1991; 10: 745-64.

13. Thorn SE, Wattwil M, Naslund I. Postoperative epidural morphine, but not epidural bupivacaine, delays gastric emptying on the first day after cholecystectomy. Reg Anesth 1992; 17: 91-4.

14. Stewart JJ, Weisbrodt NW, Burks TF. Central and peripheral actions of morphine on intestinal transit. J Pharmacol Exp Ther 1978; 205: 547-55.

15. Bredtmann RD, Herden HN, TeichmannW, et al. Epidural analgesia in colonic surgery: results of a randomized prospective study. Br J Surg 1990; 77: 638-42.

16. Ahn $\mathrm{H}$, Bronge $\mathrm{A}$, Johansson $\mathrm{K}$, et al. Effect of continuous postoperative epidural analgesia on intestinal motility. Br J Surg 1988; 75: 1176-82.

17. Carli F, Phil M, Mayo N, et al. Epidural analgesia enhances functional exercise capacity and health-related quality of life after colonic surgery. Anesthesiology 2002; 97: 540-9.

18. Holte $\mathrm{K}$, Kehlet $\mathrm{H}$. Epidural analgesia and risk of anastomotic leakage. Reg Anesth Pain Med 2001; 26: 111-7.

19. Lehman JF, Wiseman JS. Effect of epidural analgesia on the return of peristalsis and the length of stay after elective colonic surgery. Am Surg 1995; 61: 1009-12.

20. Kanazi GE, Thompso JS, Boskouski NA. Effect of epidural analgesia shortens postoperative ileus after ileal pouch-anal canal anastomosis. Am J Surg 1995; 19: 79-83.

21. Wattwil M, Thoren T, Hennerdal S, Garvill JE. Epidural analgesia with bupivacain reduces postoperative paralytic ileus after hysterectomy. Anesth Analg 1998; 68: 353-8.

22. Jansen M, Fass J, Tittel A, et al. Influence of postoperative epidural analgesia with bupivacaine on intestinal motility, transit time, and anastomotic healing. World J Surg 2002; 26: 303-6.

23. Gould TH, Grace K, Thorne G, Thomas M. Effect of thoracic epidural anaesthesia on colonic blood flow. Br J Anaesth 2002; 89 446-451. 